Research Article

\title{
Smart Localization of Thunderstorm Charge for Human 4IR Applications
}

\author{
Xu Yang $\mathbb{D}^{1,2}$ Hongyan Xing $\mathbb{D}^{1,2}$ Xinyuan Ji, ${ }^{1,2}$ and Zenghui Wang ${ }^{3}{ }^{3}$ \\ ${ }^{1}$ Collaborative Innovation Center for Meteorological Disaster Prediction and Evaluation, \\ Nanjing University of Information Science and Technology, Nanjing 210044, China \\ ${ }^{2}$ Jiangsu Key Laboratory of Meteorological Detection and Information Processing, \\ Nanjing University of Information Science and Technology, Nanjing 210044, China \\ ${ }^{3}$ Department of Electrical and Mining Engineering, University of South Africa, Florida 1710, South Africa
}

Correspondence should be addressed to Hongyan Xing; xinghy@nuist.edu.cn

Received 21 February 2021; Revised 26 March 2021; Accepted 5 April 2021; Published 16 April 2021

Academic Editor: Hoon Ko

Copyright $\odot 2021$ Xu Yang et al. This is an open access article distributed under the Creative Commons Attribution License, which permits unrestricted use, distribution, and reproduction in any medium, provided the original work is properly cited.

The relationship between smart devices and human beings is one of the research hotspots of the Fourth Industrial Revolution (4IR). In this regard, we explored the practical relationship between the $3 \mathrm{D}$ electric field components measured by the smart 3D atmospheric electric field apparatus (AEFA) and the thunderstorm activity from the perspective of the observer. Especially, in the application of AEFA, a smart calibration method is proposed to solve the problem of inconvenient thunderstorm data acquisition. Firstly, in order to obtain the thunderstorm charge position from the observation angle of the apparatus, this paper establishes a 3D electric field measurement model. According to the mirror method theory, we further obtain the charge potential distribution at AEFA. Then, the electric field components are derived by using the potential distribution formula with permittivity. In addition, based on the vector relation of the model, the thunderstorm charge azimuth and elevation angles are obtained. Finally, after the establishment of a new coordinate system, the calibration of charge localization is carried out, based on the observation point. Meanwhile, a preliminary solution is given to the problem that the elevation of the apparatus position affects the localization performance. Results show that the method matches the data of radar map and microphone array, which reflects the advantages of the method. Besides, this method can be used not only in sound source localization but also in AI thunderstorm monitoring system to realize a big data net observation.

\section{Introduction}

As one of the changes caused by the global trend of $4 \mathrm{IR}$, climate is an important research field. Thunderstorm cloud is a common weather phenomenon, which may cause serious disasters [1-3]. For buildings $[4,5]$, the metal devices with poor grounding inside are easy to produce sparks after lightning induction, which is dangerous for warehouses storing flammable products. In the case of lightning, instantaneous high currents will be introduced into the room through transmission cables, communication lines, telephone lines, and metal pipes. This may cause lightning strikes in computer networks, communication equipment, and industrial control systems due to electromagnetic induction. Through the analysis of lightning data, the incidence of such accidents is high and the consequences are very serious. According to estimates by the relevant departments, about 1,800 lightning strikes per minute around the world are in progress. Therefore, it is urgent to study thunderstorm monitoring methods with high early warning rate $[6,7]$.

The atmospheric electric field is often used to analyze thunderstorm activity, which has been widely used in meteorology, communication, and other fields [8-10]. To acquire the thunderstorm charge position, a lot of research studies have been done on the field grinding electrometer 
$[11,12]$. To determine the degree of accumulation of thunderstorm charge for lightning warning, they use the magnitude of the vertical electric field measured by AEFA. However, it is just a nondirectional detector, and it is impossible to know the specific position of the thunderstorm cloud. For example, Schumann et al. [13] analyzed the internal discharge before the positive cloud flickering to the ground. They measured the vertical component firstly. Besides, within the range of $3-80 \mathrm{~km}$ from the electric field measurement point, they found that the return stroke causes some changes in electric field characteristics. By combining the interplanetary magnetic field, Frank-Kamenetsky et al. [14] studied the relationship between the thunderstorm development and the global electric field. After a long-term research, they found that there were horizontal and vertical components $[15,16]$. Therefore, the exploration of the more real electric field is closely related to the measurement of the 3D electric field component.

From the aspects of material and structure [17-21], the researchers have improved the one-dimensional to threedimensional AEFA. Among them, Wen et al. [19] proposed a three-dimensional measurement method based on coplanar decoupling structure to solve the coupling problem between 3D atmospheric electric field components. Xu et al. [20] used particle swarm optimization (PSO) and 3D atmospheric electric field to invert the thunderstorm clouds and analyzed the electric field variation during a thunderstorm. Yang and Xing [21] presented a data complementary method to implement the network localization of thunderstorm charge. These solve the problem of being only able to measure a single electric field component by the former and reduce the electric field component coupling among existing electric field sensors. However, after using the electric field component data to obtain thunderstorm charge position information from the apparatus angle, these studies often ignore the practical significance of obtaining data from the observation angle of the apparatus.

This paper focuses on the real-time and accurate calibration of thunderstorm charge smart localization in the actual environment. Based on the classical thunderstorm detection technology, this paper focuses more on implementing omnidirectional calibration relative to the observation point. The primary reasons include the following:

(1) Classical detection techniques can only approximate the development of thunderstorm clouds. The root cause is that the classical AEFA can only measure a single vertical electric field component, which cannot meet the thunderstorm charge localization.

(2) There is a lack of 3D AEFA which can simultaneously measure horizontal and vertical electric field components. In addition, the calibration of the electric field and the neglect of permittivity measurement will affect the calibration. Therefore, it is necessary to design a smart localization fusion system.

(3) The data come from the apparatus itself, but the observation point is not necessarily in the position of the apparatus. This means the existence of real-time information dislocation. Therefore, we need to implement the angle conversion from apparatus angle to observation angle.

The contributions of this paper are as follows:

(1) In order to locate and calibrate thunderstorm charge, a $3 \mathrm{D}$ electric field measurement model is designed according to the relative position relationship between thunderstorm charge and 3D AEFA, which is different from the classical model.

(2) An all-weather calibration algorithm for the thunderstorm charge is proposed. This method focuses on the establishment of the relationship between the position of AEFA and the observation point. In other words, during the thunderstorm monitoring process, the real-time information of charge motion is obtained from the observation point.

(3) We design a fusion system for smart localization. Compared with the traditional system, the system includes 3D electric field measurement unit, electric field calibration unit, and permittivity measurement unit, which provides a guarantee for high-precision localization.

\section{Thunderstorm Charge Localization}

In this section, we establish a model of $3 \mathrm{D}$ electric field measurement and deduce the thunderstorm charge localization formula. In addition, the theory of electric field measurement is further given.

\subsection{Establishment of 3D Electric Field Measurement Model.} Based on the mirror image theory [7, 22-25], the 3D electric field measurement model for thunderstorm charge localization as shown in Figure 1 is established.

In Figure 1, $M(x, y, z)$ is the negative charge position of the lower part of the thunderstorm cloud. $N(0,0,0)$ is the position of AEFA. $h$ represents the sum of the height of the apparatus itself and the elevation of the apparatus. $(r, \alpha, \beta)$ represents the spherical coordinates of $M$, and the parameters in $(r, \alpha, \beta)$ represent the distance from $M$ to $N$, azimuth angle, and elevation angle in turn. Finally, the electric field intensity of $M$ measured by AEFA is defined as E.

Secondly, the potential distribution $\varphi$ of charge $M$ at apparatus $N$ is given by

$$
\varphi=\frac{1}{4 \pi \varepsilon_{1}}\left(\frac{q}{\sqrt{x^{2}+y^{2}+(z-h)^{2}}}-\frac{\varepsilon_{2}-\varepsilon_{1}}{\varepsilon_{2}+\varepsilon_{1}} \frac{q^{\prime}}{\sqrt{x^{2}+y^{2}+(z+h)^{2}}}\right) .
$$

In equation (1), charge $q^{\prime}$ is the mirror charge of charge q. In addition, $\varepsilon_{1}$ and $\varepsilon_{2}$ represent the permittivity of the air and the ground, respectively.

In Figure 1, the three relationships of $E_{x}, E_{y}$, and $E_{z}$ are as follows: 


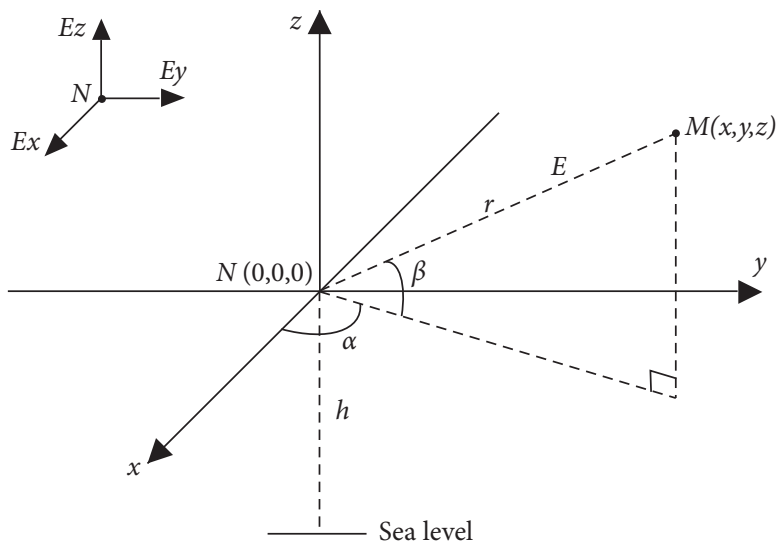

Figure 1: A 3D electric field measurement model.

$E=E_{x}+E_{y}+E_{z}$
After performing partial derivative processing on equation (1), we get $[7,22]$ :

In general, the following relationships can be obtained

$$
z \approx z-h \approx z+h
$$

It can be seen from Figure 1 that

$$
r=\sqrt{x^{2}+y^{2}+z^{2}}
$$

After substituting equation (4) into equation (3), we can get

$$
\left\{\begin{array}{l}
E_{x}=A(1-B) r^{-3} x, \\
E_{y}=A(1-B) r^{-3} y, \\
E_{z}=A(1-B) r^{-3} z .
\end{array}\right.
$$

In equation (6), $A=-q / 4 \pi \varepsilon_{1}$ and $B=\left(\varepsilon_{2}-\varepsilon_{1}\right) /\left(\varepsilon_{2}+\varepsilon_{1}\right)$. Equation (7) can be obtained by using equation (6):

$$
\left\{\begin{array}{l}
r=\sqrt[4]{\frac{A^{2}(1-B)^{2}}{E_{x}^{2}+E_{y}^{2}+E_{z}^{2}}}, \\
\alpha=\arctan \frac{E_{y}}{E_{x}} \\
\beta=\arctan \frac{\varepsilon_{1} E_{z}}{\varepsilon_{2} \sqrt{E_{x}^{2}+E_{y}^{2}}} .
\end{array}\right.
$$

Finally, according to equation (7), $M(x, y, z)$ can be further obtained as follows:

$$
\left\{\begin{array}{l}
x=r \cos \alpha \cos \beta, \\
y=r \sin \alpha \cos \beta, \\
z=r \sin \beta .
\end{array}\right.
$$

It can be seen from Figure 1 that when the thunderstorm cloud appears above the electric field apparatus, a large amount of charge will accumulate in the cloud. At this time, the electric field $E$ generated by the thunderstorm charge $M$ will be sensed by the 3D electric field sensor of the apparatus. After that, the coordinates of the thunderstorm charge can be obtained by calculation, which can provide a data source for locating the charge. By contrast, the classical thunderstorm detection model is mainly based on the field mill type atmospheric electric field meter, as shown in Figure $2[11,12]$.

In Figure 2, the point $S$ represents the position of the field mill type electric field meter. Different from Figure 1, after the vertical electric field component is obtained by using this model, only the distance between charge $M$ and point $S$ and the degree of charge accumulation in the cloud can be roughly judged. In practical application, the position of the thunderstorm charge cannot be determined by using a onedimensional component, which leads to its poor thunderstorm warning ability. Figure 3 shows the difference between the two. 


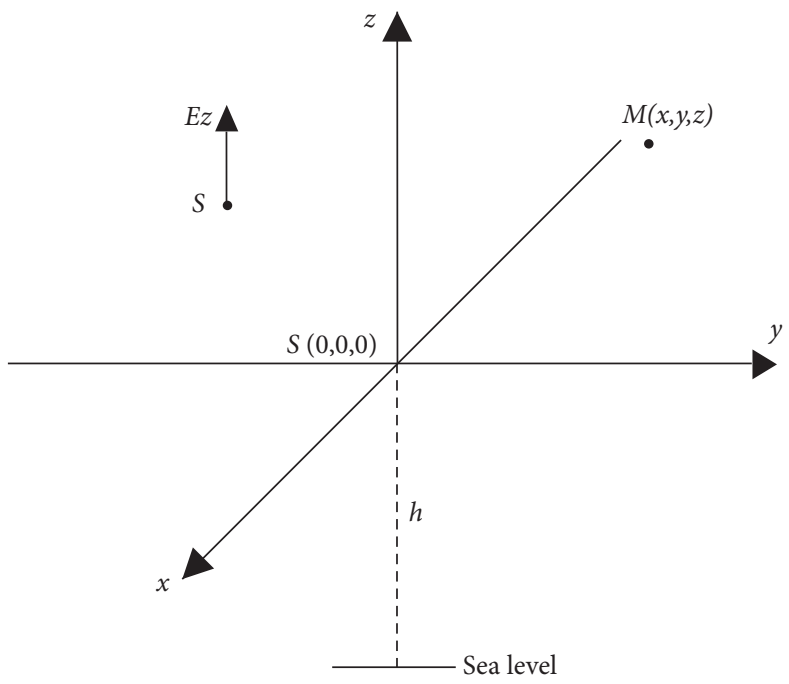

FIgURE 2: Classical model of atmospheric electric field measurement.

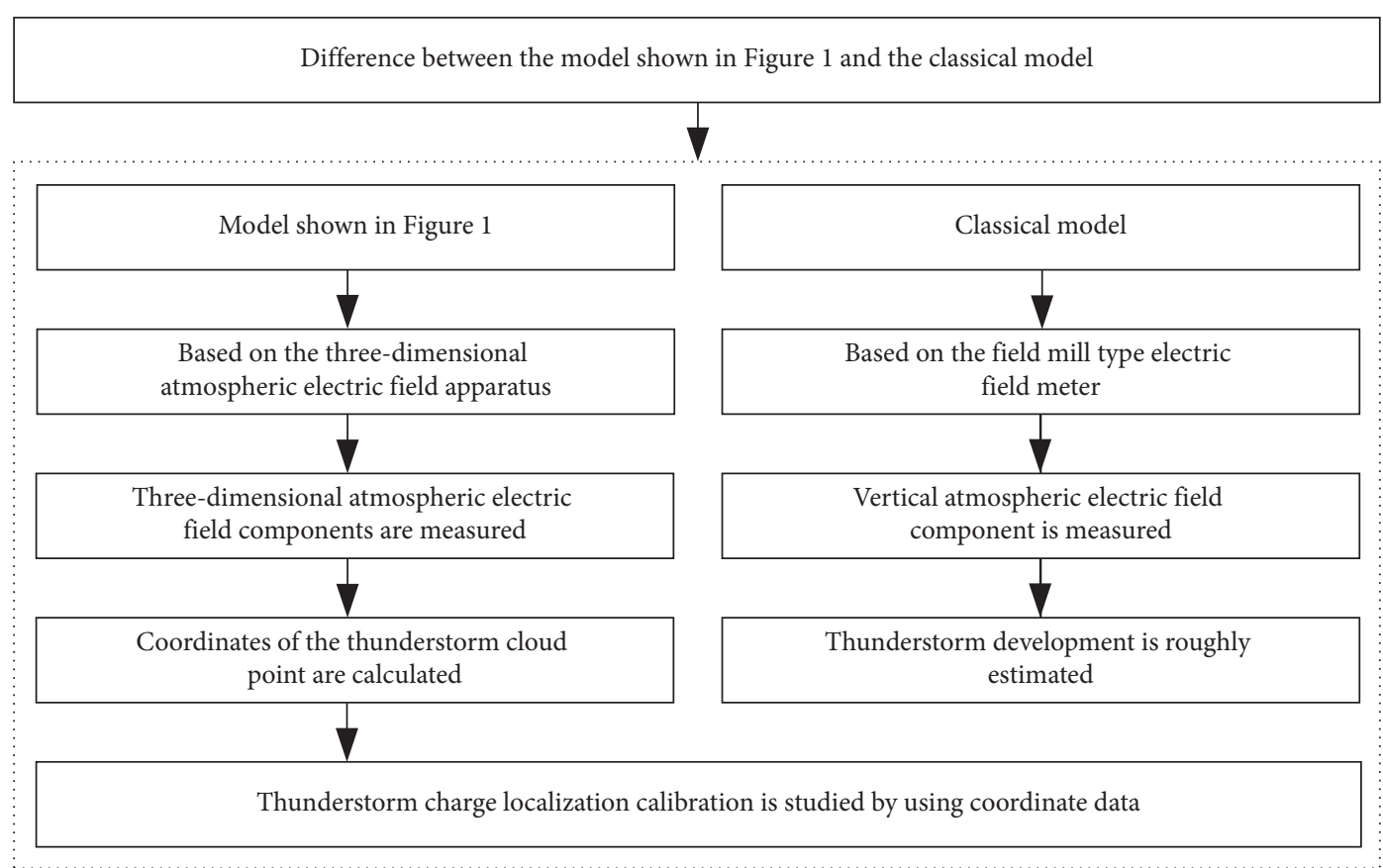

FIgURE 3: Comparison between the model shown in Figure 1 and the classical model.

2.2. Principle of 3D Electric Field Measurement. The measurement of the 3D atmospheric electric field is the basis of smart localization. After analyzing the advantages and disadvantages of AEFA [7, 8, 19-22], a 3D atmospheric electric field sensor structure is designed for atmospheric electric field measurement. Details are shown in Figure 4.

In Figure 4, the smart single-axis rotary vane atmospheric electric field sensor is mainly composed of a rotating blade, a shielded rotor, a photoelectric switch, a synchronous blade, a motor, an induction electrode $Z$, and an induction electrode $X$, and $Y$ surrounding and parallel to the $Z$-axis. Among them, there are 8 sensing slices in both the horizontal direction and the vertical direction, and their arrangement positions are shown in Figure 5.
In Figure 5(a), there are 8 sensing slices in the vertical electrode direction. Divide these sensing slices into two groups, each with four slices, and alternately arrange them in a fan shape. At the same time, the electrodes with the same name are electrically connected, that is, $Z a$ and $Z b$ are connected separately. In this way, when one set of sensing slices is shielded by the shielded rotor, the other set of sensing slices will be exposed to the electric field to form a differential signal.

In Figure 5(b), there are also 8 sensing slices in the horizontal electrode direction, which are divided into four groups, each with two slices. Among them, the horizontal electrodes are divided into $X$-direction electrodes and $Y$ direction electrodes. The electrodes in each direction are 


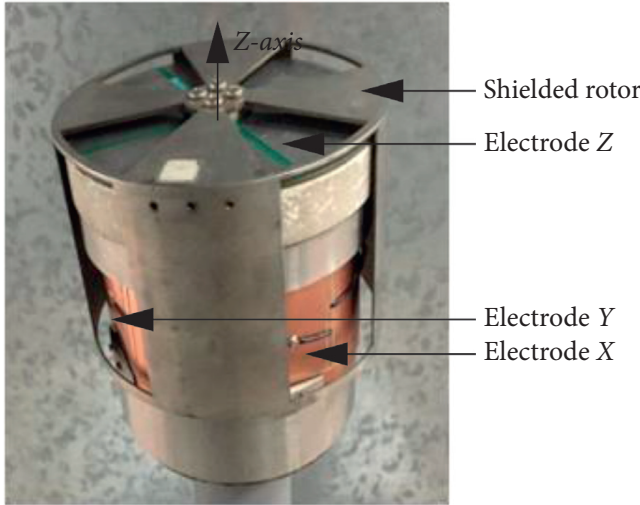

(a)

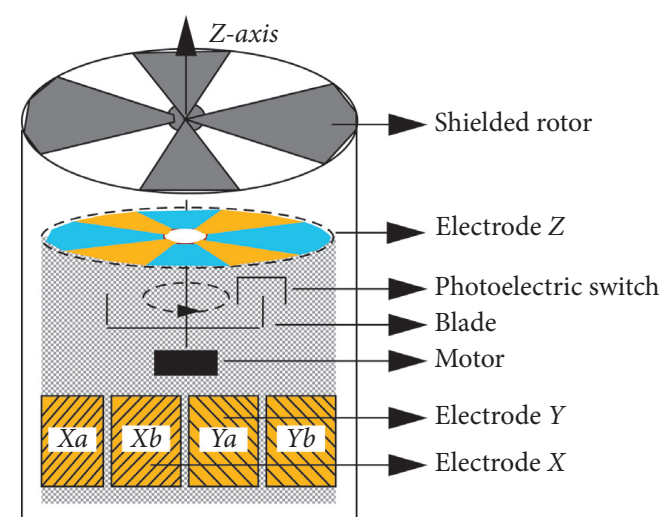

(b)

FIgURE 4: (a) The photograph and (b) the schematic of the 3D atmospheric electric field sensor.

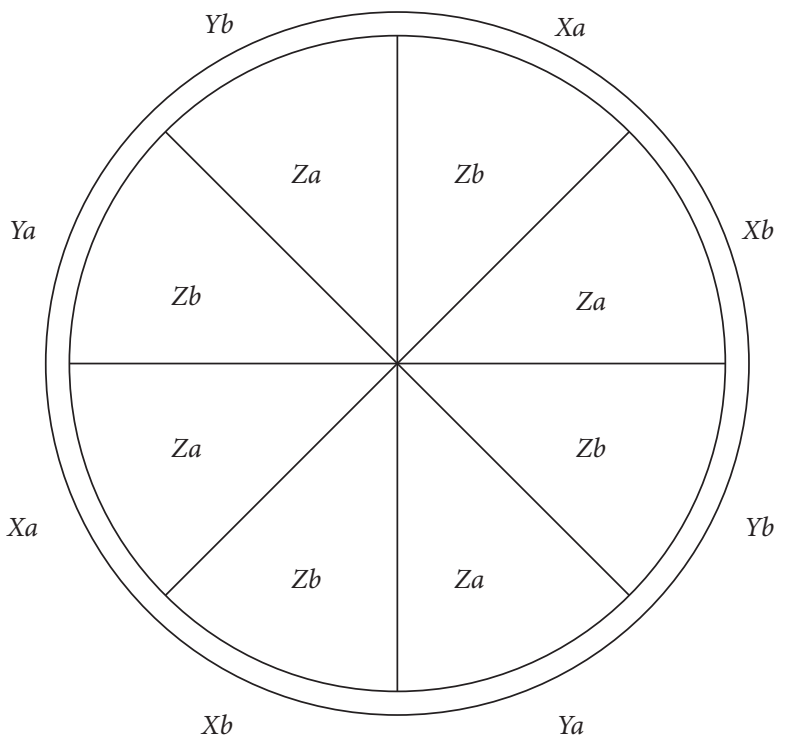

(a)

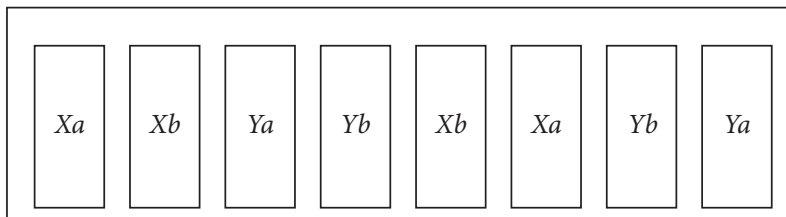

(b)

FIGURE 5: Distribution diagram of the sensing slice of the 3D atmospheric electric field sensor. (a) Distribution of the sensing slice in the direction of the vertical electrode and (b) distribution of the sensing slice in the direction of the horizontal electrode.

divided into two groups, $a$ and $b$. The electrodes with the same name are electrically connected, that is, the two pieces of $X a, X b, Y a$, and $Y b$ are connected, respectively. After that, when one set of sensing slices is exposed to the horizontal electric field and the other set is shielded by the shielded rotor, the two sets of electrodes will form currents of opposite polarity, thus forming a pair of differential signals. In addition, the photoelectric switch is fixed on the back of the $Z$-direction electrode, and the hollow-shaped small blade without a fixed ring rotates synchronously with the shielded rotor.

The outer layer of the 3D atmospheric electric field sensor is the shielding electrode, and its upper surface and side surfaces are hollow cylindrical metal slices. Specifically, there are 4 symmetrical hollowed-out sectors on the upper surface with 45 degrees and 4 symmetrical hollowed-out side cylindrical surfaces with 45 degrees on the circumference. At the same time, the hollow shape corresponds to the distribution of the sensing electrodes. Both the upper surface of the shielding electrode and the lower edge of the side are equipped with fixed rings to avoid vibration or deformation during rotation. When AEFA is working, the grounded shielding electrode on the sensor rotates under the drive of the motor to periodically shield the sensing electrode. At this time, a variable induced charge is generated on the sensing electrode, thereby forming 3 sets of alternating current signals, which are then, respectively, connected to the subsequent circuits.

The electrodes in all three directions are composed of two symmetrical metal pieces, which can generate differential induced currents and suppress common-mode noise. The $X$ and $Y$ electrodes are each composed of two rectangular metal sheets distributed at opposite positions on the sides of the cylinder. The chord width of each metal piece is 
equal to $1 / 8$ of the arc length of the entire circumference. The $Z$ electrode is composed of two metal sheets, light blue A and orange $\mathrm{B}$. The shielding cover rotation frequency is $f$, and the induced charges are $q_{x}(t), q_{y}(t)$, and $q_{z}(t)$. The area of a single metal piece of the electrodes $X$ and $Y$ is $S$. The inner and outer radii of the $Z$ electrode disk are $r_{1}$ and $r_{2}$, respectively. With the rotation of the shield, the three sensing electrodes are gradually exposed from time 0 , and the electrodes are completely exposed at time $T / 2$. During the time $(T / 2)-T$, the three electrodes are gradually blocked by the shield. Then, the induced currents $i_{x(y)}(t)$ and $i_{z}(t)$, generated by one of the metal pieces of electrode $X(Y)$ and by the $A$ portion of the electrode $Z$, respectively, are

$$
\begin{cases}i_{z}(t)=\frac{\mathrm{d} q_{z}(t)}{\mathrm{d} t}= \begin{cases}-4 \pi \varepsilon_{1} E_{z} f\left(r_{2}^{2}-r_{1}^{2}\right), & t \in\left(0, \frac{T}{2}\right), \\ 4 \pi \varepsilon_{1} E_{z} f\left(r_{2}^{2}-r_{1}^{2}\right), & t \in\left(\frac{T}{2}, T\right),\end{cases} \\ i_{x(y)}(t)=\frac{\mathrm{d} q_{x(y)}(t)}{\mathrm{d} t}= \begin{cases}-\sqrt{2} \pi \varepsilon_{1} E_{X(Y)} S f \cos \left(\frac{\pi}{4}-2 \pi f t\right), & t \in\left(0, \frac{T}{2}\right), \\ \sqrt{2} \pi \varepsilon_{1} E_{X(Y)} S f \cos \left(\frac{\pi}{4}-2 \pi f t\right), & t \in\left(\frac{T}{2}, T\right) .\end{cases} \end{cases}
$$

In equation (9), when the frequency $f$ is constant, the induced current generated by electrodes $X, Y$, and $Z$ has a linear relationship with the external electric field. It should be noted that the periods of the induced currents in three directions are the same. The electric field intensity can be determined by measuring the induced voltage.

\section{Analysis of Thunderstorm Charge Localization Performance}

In this section, after determining the relationship between the localization parameters and localization error, we further analyze the localization performance. The thunderstorm charge localization performance is related to the measurement error of electric field components $E_{x}, E_{y}$, and $E_{z}$, charge amount $q$, and permittivities $\varepsilon_{1}$ and $\varepsilon_{2}$. When charge amount $q$ and the two permittivities above are constant, the measurement accuracy of the electric field components plays a key role in the localization performance.

Let $\sigma_{E_{k}}$ be the standard deviation of the components, $k=x, y, z$, and they are equal to $\sigma_{E_{i}}[26,27]$. In addition, let us take the permittivities $\varepsilon_{1}$ and $\varepsilon_{2}$ to be equal to 1 and 5, respectively $[20,23,24,26,27]$.

After using equation (7), the measurement errors of $r, \alpha$, and $\beta$ caused by error $\sigma_{E_{i}}$ are $[25,28,29]$

$$
\left\{\begin{array}{l}
\sigma_{r E_{i}}=\frac{6}{5} \pi^{3 / 2} r^{3} \sigma_{E_{i}}, \\
\sigma_{\alpha E_{i}}=\frac{12}{5} \pi r^{2} \sqrt{1+25 \tan ^{2} \beta} \sigma_{E_{i}}, \\
\sigma_{\beta E_{i}}=\frac{12}{25} \pi r^{2} \cos \beta \sqrt{\left(1+24 \sin ^{2} \beta\right)\left(1+25 \tan ^{2} \beta\right)} \sigma_{E_{i}} .
\end{array}\right.
$$

In equation (10), $\sigma_{r E_{i}}, \sigma_{\alpha E_{i}}$, and $\sigma_{\beta E_{i}}$, respectively, are the measurement errors of $r, \alpha$, and $\beta$. In particular, the reduction of error $\sigma_{E_{i}}$ can effectively improve the localization performance.

3.1. Analysis of Ranging Performance. According to equation (10), the error curve in Figure 6 can be obtained.

In Figure 6, ranging error $\sigma_{r E_{i}}$ is affected by distance $r$ and error $\sigma_{E_{i}}$ and is greatly affected by the former. Ranging error $\sigma_{r E_{i}}$ increases as distance $r$ and error $\sigma_{E_{i}}$ increase. When error $\sigma_{E_{i}}$ is between 0 and $1 \mathrm{kV} / \mathrm{m}$, error $\sigma_{r E_{i}}$ is almost independent of distance $r$ variation. Meanwhile, when $\sigma_{E_{i}}$ is between 1 and $2 \mathrm{kV} / \mathrm{m}$, error $\sigma_{r E_{i}}$ increases sharply as distance $r$ increases. In summary, error $\sigma_{r E_{i}}$ is less than $0.12 \mathrm{~km}$, which is acceptable.

3.2. Analysis of Direction-Finding Performance. The simulation results of the direction-finding error are obtained by using equation (10), as shown in Figures 7 and 8.

In Figure 7, the azimuth angle measurement error $\sigma_{\alpha E_{i}}$ increases with the increase of distance $r$ and elevation angle $\beta$. Among them, when $r$ is less than $1 \mathrm{~km}$, error $\sigma_{\alpha E_{i}}$ is almost unaffected by the change of elevation angle $\beta$, and the maximum error $\sigma_{\alpha E_{i}}$ is only 0.5 degrees. In contrast, when $r$ is greater than $1 \mathrm{~km}$, as angle $\beta$ increases, the maximum error $\sigma_{\alpha E_{i}}$ increases to 1.9 degrees.

In Figure 8, with the increase in distance $r$ and elevation angle $\beta$, the elevation angle measurement error $\sigma_{\beta E_{i}}$ increases slowly. It is worth noting that when $r$ is between $1 \mathrm{~km}$ and $2 \mathrm{~km}$, as elevation angle $\beta$ increases, error $\sigma_{\beta E_{i}}$ slowly climbs to 0.16 degrees.

\section{Thunderstorm Charge Localization Calibration}

In this section, in order to obtain the thunderstorm charge position directly from the observation point, we establish a 


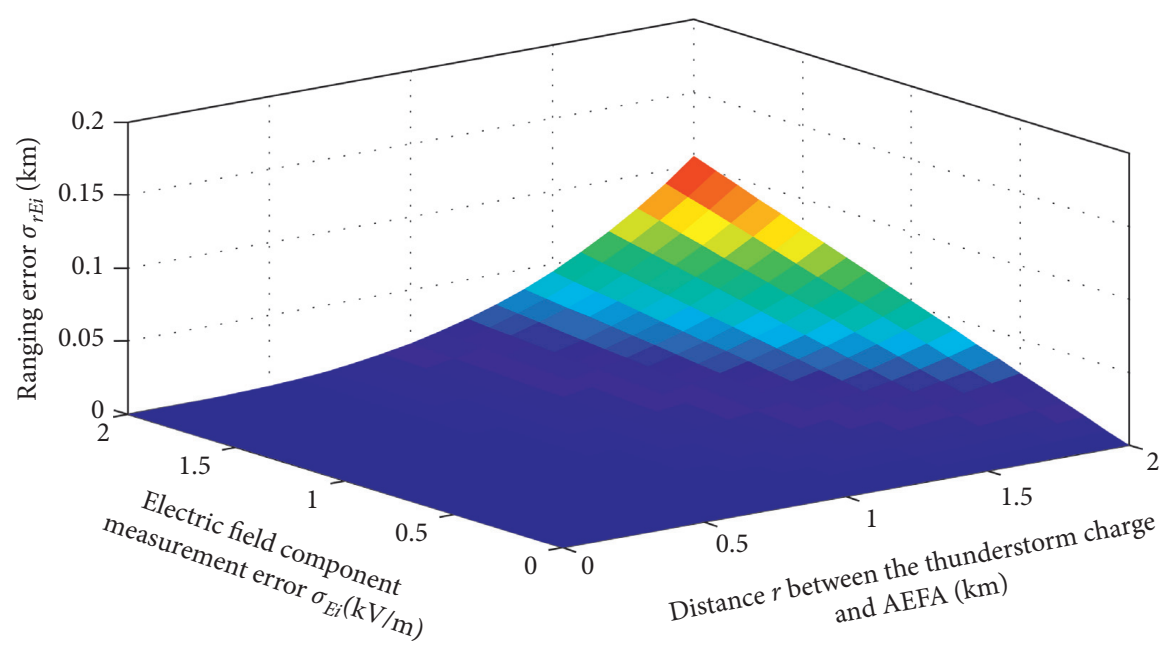

FIGURE 6: Analysis chart of ranging error.

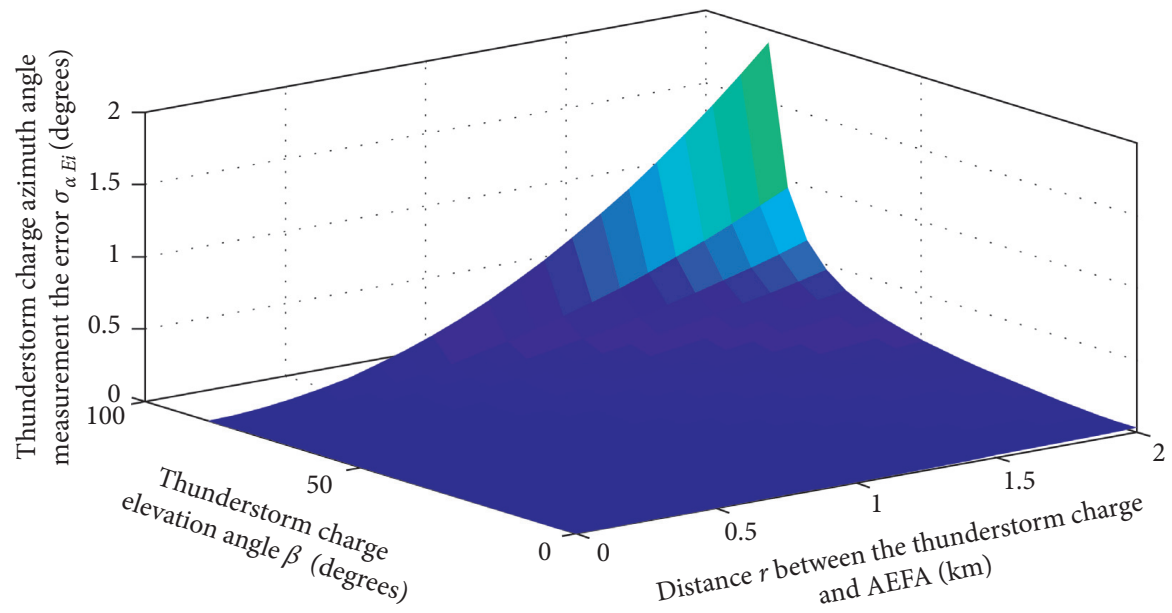

Figure 7: Analysis chart of azimuth angle measurement error.

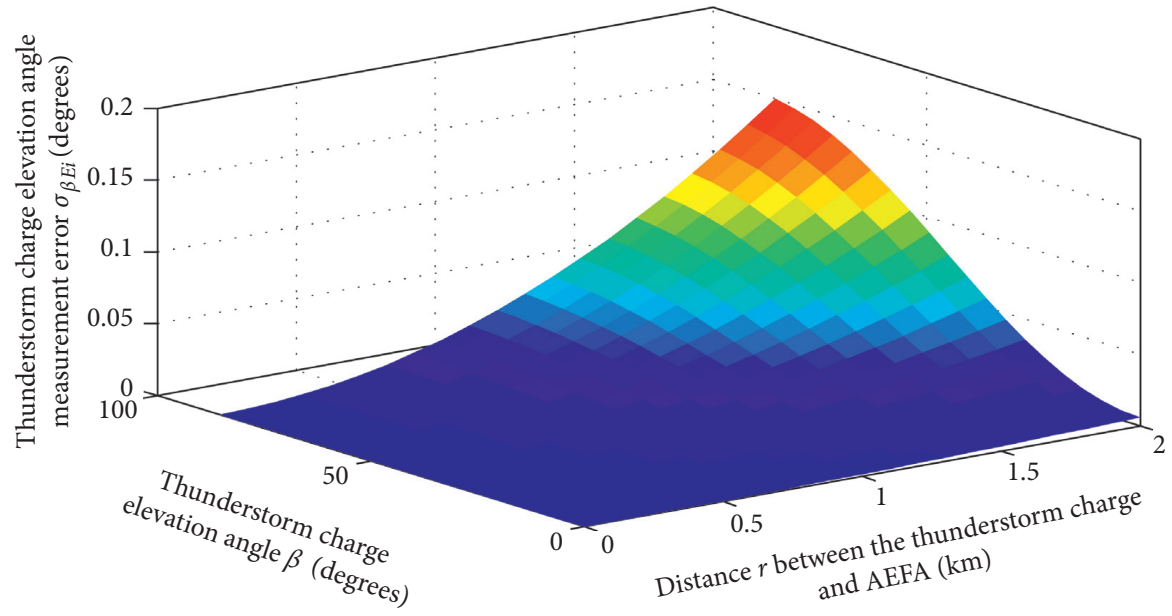

Figure 8: Analysis chart of elevation angle measurement error. 
charge localization calibration model and propose its calibration method. In addition, an elevation calibration method is given preliminarily. In the actual environment, the thunderstorm charge localization cannot be intuitively transmitted to its position information, based on AEFA. To realize the calibration of charge localization, we firstly use the apparatus to measure the thunderstorm charge orientation data. Then, the spatial rectangular coordinate system is reestablished based on the observation angle of AEFA. Finally, we introduce a calibration method to achieve the above purpose.

4.1. Thunderstorm Charge Localization Calibration Model. Let observation angle $p$ of the electric field apparatus be located in the original coordinate system, and substitute the coordinates of $p$ for $(X, Y, Z)$. Based on the observation angle of AEFA, the localization calibration model is shown in Figure 9.

According to Figure 9, a new coordinate system is established with observation point $P(X, Y, Z)$ as the origin, and $(X, Y, Z)$ is converted into $(0,0,0)$. Based on the coordinate system of $p$, thunderstorm charge position $M(x, y, z)$ measured by the apparatus $N(0,0,0)$ can be converted into $(x-X, y-Y, z-Z)$, and it is equivalent to $M\left(X^{\prime}, Y^{\prime}, Z^{\prime}\right)$. Let the thunderstorm charge azimuth angle relative to the observation angle be $\theta$, its elevation angle be $\psi$, and its distance from charge $M$ to observation point $p$ be $R^{\prime}$.

4.2. Thunderstorm Charge Localization Calibration Method. According to the model shown in Figure 9, the localization calibration is performed, and the corrected thunderstorm charge coordinates are

$$
\left\{\begin{array}{l}
X^{\prime}=x-X \\
Y^{\prime}=y-Y \\
Z^{\prime}=z-Z \\
R^{\prime}=\sqrt{X^{\prime 2}+Y^{\prime 2}+Z^{\prime 2}}
\end{array}\right.
$$

In Figure 9, the azimuth and elevation angles $\theta$ and $\psi$ are obtained by using the solid geometry analysis method [30-32]:

$$
\left\{\begin{array}{l}
\theta=\arctan \frac{Y^{\prime}}{X^{\prime}}, \\
\psi=\arcsin \frac{Z^{\prime}}{R^{\prime}} .
\end{array}\right.
$$

For any spatial position of thunderstorm charge $M$, if we combine equations (11) and (12), the localization calibration can be realized.

In fact, it is also necessary to study the elevation calibration where the $3 \mathrm{D}$ AEFA is located. Generally speaking, the height of the thunderstorm charge from sea level is two orders of magnitude higher than the elevation where the

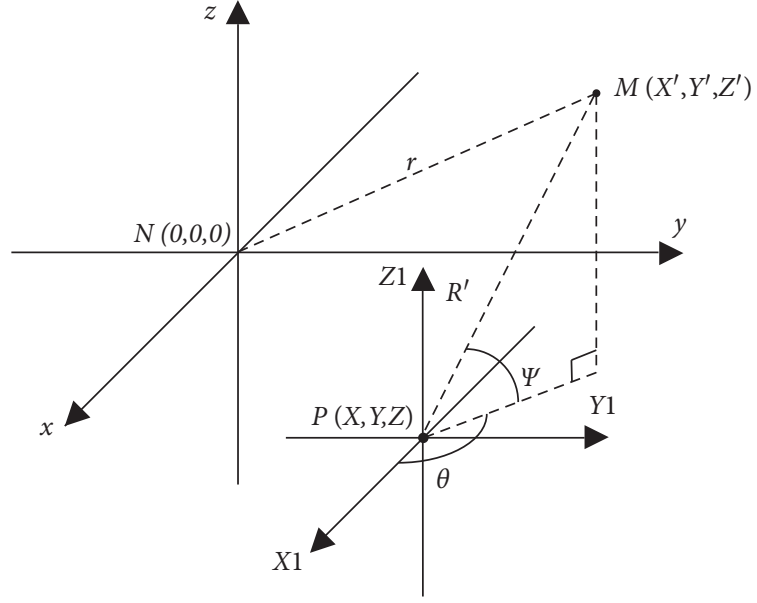

Figure 9: Model of the thunderstorm charge localization calibration.

AEFA is located. Therefore, when the thunderstorm charge localization is conducted, the AEFA is usually regarded as the coordinate system origin where the charge is located. In fact, the origin should be the intersection of the $Z$-axis where AEFA is located and the sea level. The elevation where each apparatus is installed is different, so the data without elevation calibration are very likely to directly affect the reliability of the localization result. To this end, the model of elevation calibration is shown in Figure 10.

In addition to having the parameter characteristics of the model shown in Figure 1, in Figure 10, the elevation calibration point $S(0,0,-h)$ is the intersection of the $Z$-axis where the apparatus is located and the sea level, and the $3 \mathrm{D}$ electric field components after calibration are $E_{x}^{\prime}, E_{y}^{\prime}$, and $E_{z}^{\prime}$. The corrected horizontal angle and elevation angle of the charge are $\alpha^{\prime}$ and $\beta^{\prime}$, respectively. $r^{\prime}$ is the distance from $M$ to $S$. The electric field intensity of $M$ at the calibration point $S$ is $E^{\prime}$.

Based on the model shown in Figure 10, the corrected 3D electric field components $E_{x}^{\prime}, E_{y}^{\prime}$, and $E_{z}^{\prime}$ are

$$
\left\{\begin{array}{l}
E_{x}^{\prime}=E_{x}, \\
E_{y}^{\prime}=E_{y}, \\
E_{x}^{\prime}=E^{\prime} \cdot \sin \beta^{\prime}=E^{\prime} \cdot \frac{z+h}{r^{\prime}} .
\end{array}\right.
$$

From equation (13), we can get

$$
\left\{\begin{array}{l}
E_{x}^{\prime}=E_{x}, \\
E_{y}^{\prime}=E_{y}, \\
E_{z}^{\prime}=\sqrt{E_{x}^{2}+E_{y}^{2}} \cdot C .
\end{array}\right.
$$

Among them, $C=(z+h) / \sqrt{x^{2}+y^{2}}$. The thunderstorm charge spherical coordinates $M\left(r^{\prime}, \alpha^{\prime}, \beta^{\prime}\right)$ after elevation calibration obtained by equation (14) are 


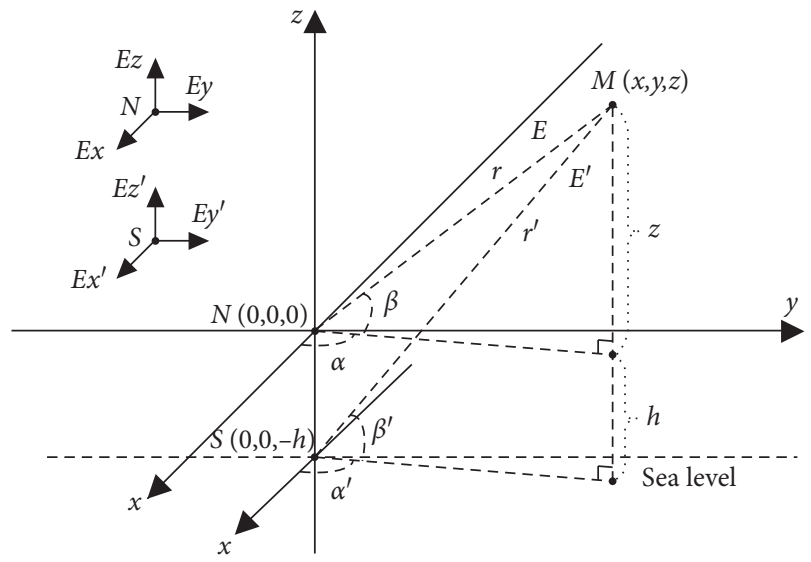

FIgURE 10: A elevation calibration model for thunderstorm charge localization.

$$
\left\{\begin{array}{l}
r^{\prime}=\sqrt[4]{\frac{A^{2}(1-B)^{2}}{\left(1+C^{2}\right)\left(E_{x}^{2}+E_{y}^{2}\right)},} \\
\alpha^{\prime}=\arctan \frac{E_{y}}{E_{x}}, \\
\beta^{\prime}=\arctan \frac{\varepsilon_{1}}{\varepsilon_{2}} C .
\end{array}\right.
$$

From equation (15), it can be preliminarily seen that there is a certain relationship between the elevation $h$ and the error of thunderstorm charge localization. Here, the validation of the elevation calibration method and the comparison with related technologies will be studied in the future work.

Finally, the flowchart of the smart calibration method as shown in Figure 11 is given.

\section{Structure Design of Smart Localization}

In order to achieve smart localization, a fusion system is obtained by combining 3D electric field measurement unit, electric field calibration unit, and permittivity measurement unit. The schematic diagram is shown in Figure 12.

In Figure 12, after the electric field calibration is carried out, the two sensors first convert physical signals into electrical signals. Then, through signal conditioning and photoelectric conversion, the electrical signals are converted into digital signals. Furthermore, the signals are sent to the main control system for processing. After introducing the smart localization method, the performance of the method is analyzed. Finally, the effectiveness of the method is verified by the actual experiments in different weather conditions. In addition to the electric field measurement unit given in Section 2.2, the following three parts are included: electric field calibration unit, permittivity measurement unit, and system performance parameters.
5.1. Electric Field Calibration Unit. To ensure the accuracy of measured electric field data, we implement the calibration of the electric field apparatus before the experiments. Building a known standard uniform electric field is the key to effective calibration. For this, a uniform electric field is generated by using two parallel plate capacitors as shown in Figure 13.

In Figure 13, the upper and lower parallel electrode plates $C_{1}$ and $C_{2}$ are both square thin metal plates, and the distance between the two plates is $2 d$. The electric field apparatus $N$ is located on the center line $L$ between the two parallel plates, and the distance from its center position to the plate edge is $D$. After adding DC voltages of $V_{0}$ and $-V_{0}$ to the two plates, the uniform electric field value generated between the two plates is given by

$$
E=\frac{V_{0}-\left(-V_{0}\right)}{2 d}=\frac{V_{0}}{d} .
$$

After voltages $V_{0}$ and $-V_{0}$ are input into the parallel electrode plates, uniform electric fields of different intensity are generated by adjusting the voltages. On the one hand, after the real-time voltage value is measured by the data collector, the theoretical value of the electric field intensity can be calculated between the plates, according to distance $2 d$ between the plates. On the other hand, an electric field sensor is used to convert the electric field value between the plates into a direct current voltage signal. Finally, by comparing the theoretical and measured values, the calibration of the electric field can be achieved.

5.2. Permittivity Measurement Unit. Considering the inconvenience of sampling and real-time measurement, a device which can ensure the measurement accuracy of the permittivity contained in equation (1) is put forward by using coplanar electrode sensor. The equivalent circuit of the device is shown in Figure 14.

In Figure $14, \varepsilon_{r}$ represents the permittivity. The device mainly includes the coplanar capacity sensor with 4 electrodes. Among them, electrodes 1 and 2 are the measurement electrodes and electrodes 3 and 4 are the shielding electrodes. The latter two electrodes are connected to the ground to shield the interference of the environmental electric field. The capacitance $C_{x}$ is the measured capacitance between electrode 1 and electrode 2 . On the one hand, the difference $\Delta C$ of the capacitance $C_{x}$ and $C_{\text {air }}$ is the linear function of measuring material permittivity. On the other hand, the capacitance values $C_{12}, C_{13}$, and $C_{23}$ are fixed and can be eliminated during measurement. Therefore, the permittivity $\varepsilon_{r}$ can be calculated as follows:

$$
\varepsilon_{r}=\frac{\Delta C}{C_{\text {air }}}+1
$$

5.3. Performance Parameter. The smart localization system can locate the thunderstorm charge with a radius of $15 \mathrm{~km}$, and the error of ranging and direction finding is less than $10 \%$. The specific performance parameters are shown in Table 1. 


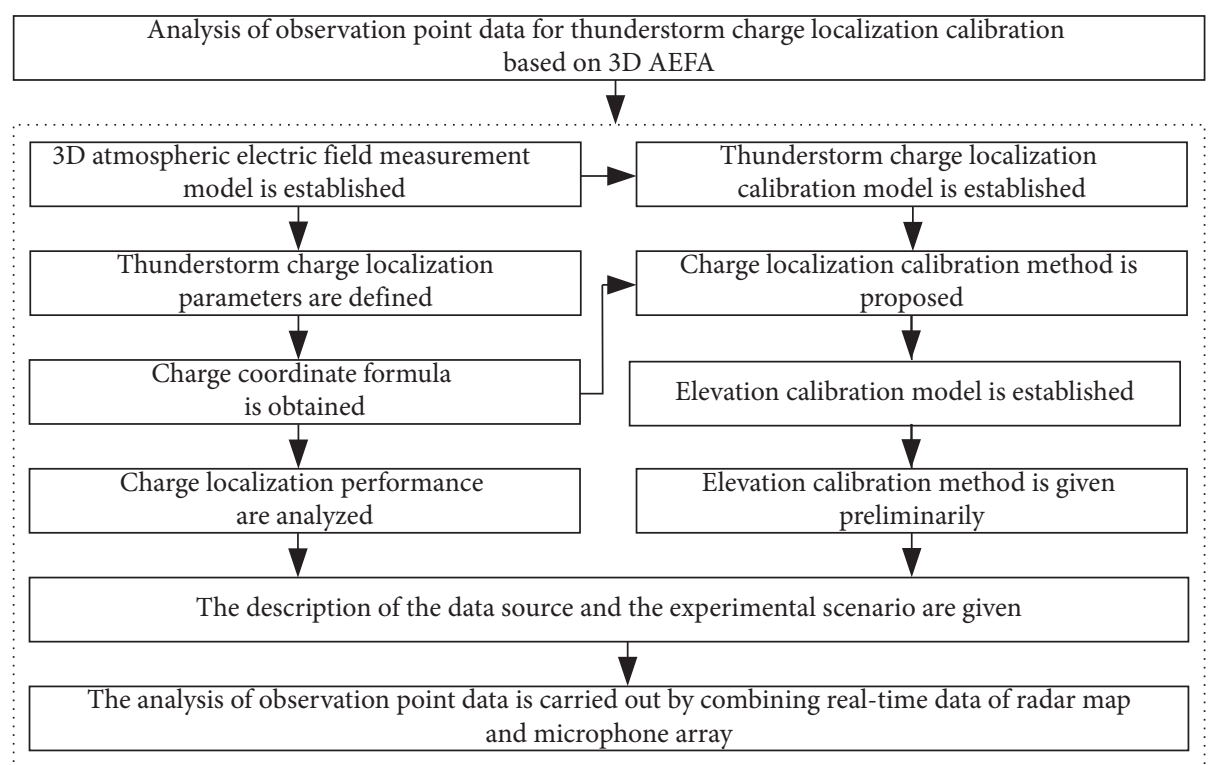

Figure 11: The flowchart of the thunderstorm charge localization calibration.

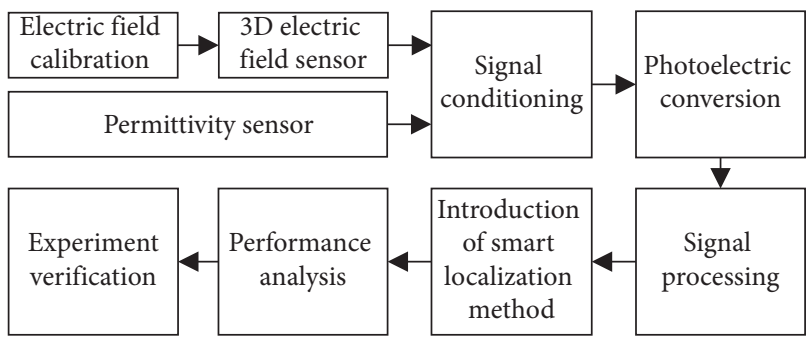

FIgURE 12: A schematic diagram of the system framework.

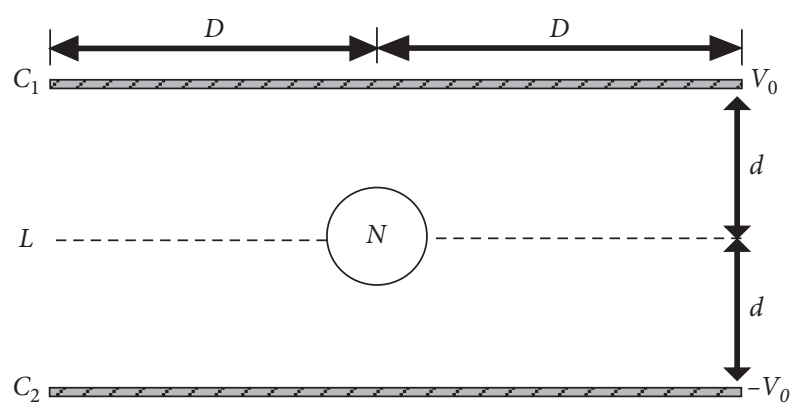

FIgURe 13: Calibration diagram of electric field.

\section{Experimental Measurement Results and Analyses}

In Figure 15, we install the smart 3D AEFA on the roof of the School of Electronics and Information Engineering, Nanjing University of Information Science and Technology (NUIST). The apparatus is located about 28 meters above sea level. The $3 \mathrm{D}$ electric field components $\left(E_{x}, E_{y}, E_{z}\right)$ (units: $\left.\mathrm{kV} / \mathrm{m}\right)$ are recorded by a computer to obtain thunderstorm charge position $M\left(r^{\prime}, \alpha^{\prime}, \beta^{\prime}\right)$ (units: $\mathrm{km}$, degrees, degrees).

As shown in Figure 15, AEFA and microphone array [21] are used for comparative experiments, and the latter

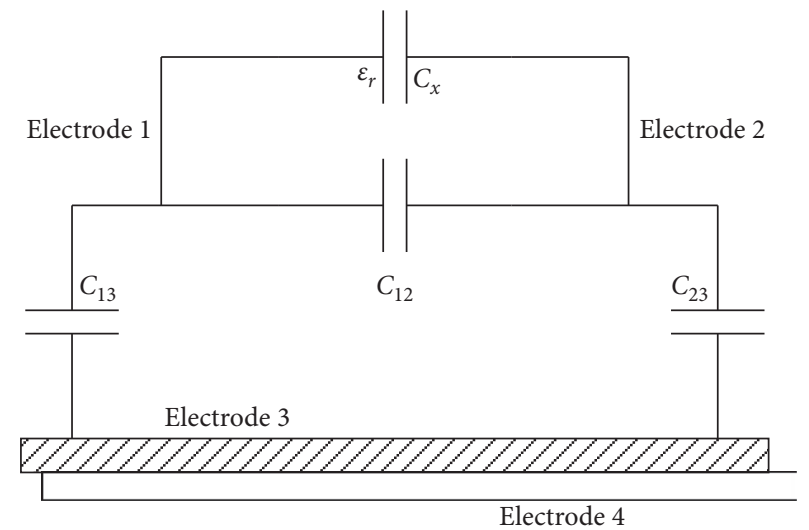

FIGURE 14: Equivalent circuit of permittivity measurement device.

TABLE 1: Smart system performance parameters.

\begin{tabular}{lc}
\hline Parameter type & Specific value \\
\hline Power & DC voltage $12 \mathrm{~V}$ \\
Electricity consumption & $<15 \mathrm{~W}$ \\
Temperature and humidity & $-25 \sim 85^{\circ} \mathrm{C}, 0 \sim 100 \% \mathrm{RH}$ \\
Measurement radius & $<15 \mathrm{~km}$ \\
Electric field measurement range & $-50 \mathrm{kV} / \mathrm{m} \sim 50 \mathrm{kV} / \mathrm{m}$ \\
Direction-finding error & $<10 \%$ \\
False alarm rate & $<10 \%$ \\
\hline
\end{tabular}

used to locate possible thunder source. Figure 15(c) shows the schematic diagram of AEFA $N$ and the array center $N 1$ being placed at the same point. In the actual environment, AEFA should be placed within 5 meters from the array center. It should be noted that the elevation of the shielded rotor plane on the top of the electric field sensor should be equal to that of the $X O Y$ plane in the array. Here, the positive half of the $x$-axis and the $y$-axis of the new coordinate system are adjusted to the due south and the due 


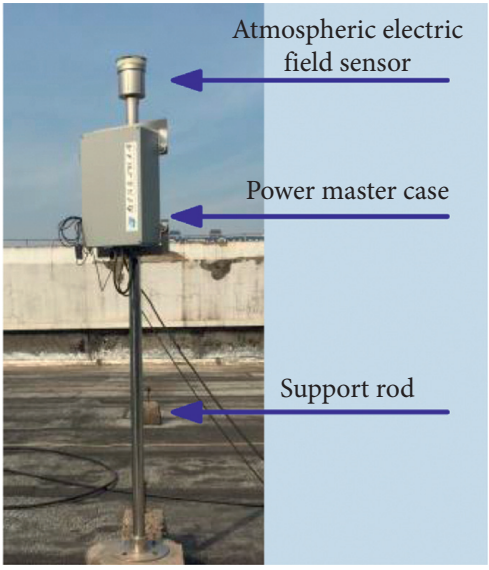

(a)

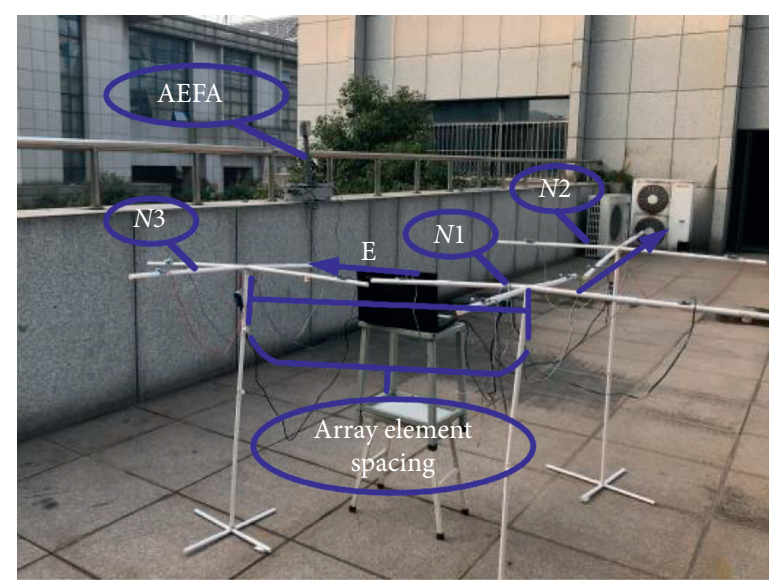

(b)

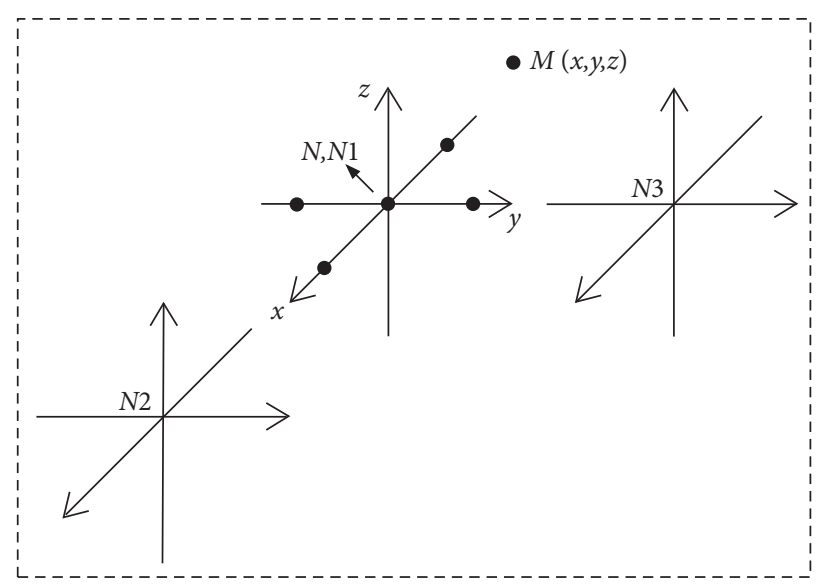

(c)

Figure 15: The installation of the smart 3D AEFA and the microphone array. (a) 3D AEFA, (b) microphone array, and (c) placement diagram of 3D AEFA and microphone array.

east directions, respectively. At the same time, the direction of the microphone array is adjusted in the same way.

Before the experiments, by using the complementary ensemble empirical mode decomposition with adaptive noise and Savitzky-Golay filtering (CEEMDAN-SG), the atmospheric electric field signal collected by AEFA is denoised [21]. More specifically, after CEEMDAN is used to decompose the electric field signal into a series of intrinsic mode function components, the signal is reconstructed after SG filtering of those noise-dominant components. After the observation angle $p$ is set as $(0.03,0.04,-0.01)$ (units: $\mathrm{km}$ ), the following experiments are conducted, respectively.

6.1. Experiments in Thunderstorm Weather. At 10:11 on April 9, 2019, the results of AEFA and microphone array are shown in Table 2. Meanwhile, Figure 16 shows the radar map of Nanjing radar station. The red cross marked in the radar map is the observation point.

In Table 2, the thunderstorm charge coordinates of the observation point are $(0.438,-86.97,71.09)$ (unit: $\mathrm{km}$, degrees, degrees). The thunderstorm charge is 86.97 degrees south by west and $0.438 \mathrm{~km}$ away from the observation point. It is worth noting that the elevation angle is 71.09 degrees. Therefore, it can be inferred that the thunderstorm cloud already exists above the observation point. In combination with Figure 16, the fact that radar echo intensity is over $45 \mathrm{dBZ}$ indicates that a large amount of charge has accumulated in the cloud above the observation point, reflecting the existence of thunderstorm. To sum up, at 10:11 on April 9, 2019, the calibration method is consistent with the radar map data, which indicates that the thunderstorm cloud appears above the observation point. This shows the feasibility of the method.

On the premise that it is feasible to obtain the thunder source position from the microphone array, in order to compare the calibration effect with Ref. [29], the observation point we select for both is $(0.03,0.04,-0.01)$ (units: $\mathrm{km}, \mathrm{km}$, $\mathrm{km}$ ). In addition, the array element spacing is set to $0.8 \mathrm{~m}$. At the same time point, the measured results are shown in Table 2.

In Table 2, from the perspective of array, the sound source point coordinates are $(0.0072,-0.1368,0.4254)$ (units: $\mathrm{km}$ ). The sound source is about 86.99 degrees south by west and $0.447 \mathrm{~km}$ away from the array center. Besides, its elevation angle is 72.15 degrees, which is almost 
TABLE 2: Experimental results in thunderstorm weather.

\begin{tabular}{lc}
\hline Data source & Results \\
\hline Electric field components & $(0.014,-0.038,0.755)$ \\
AEFA angle & $(0.419,-69.78,74.99)$ \\
Observation angle & $(0.438,-86.97,71.09)$ \\
Microphone array & $(0.0072,-0.1368,0.4254)$ \\
\hline
\end{tabular}

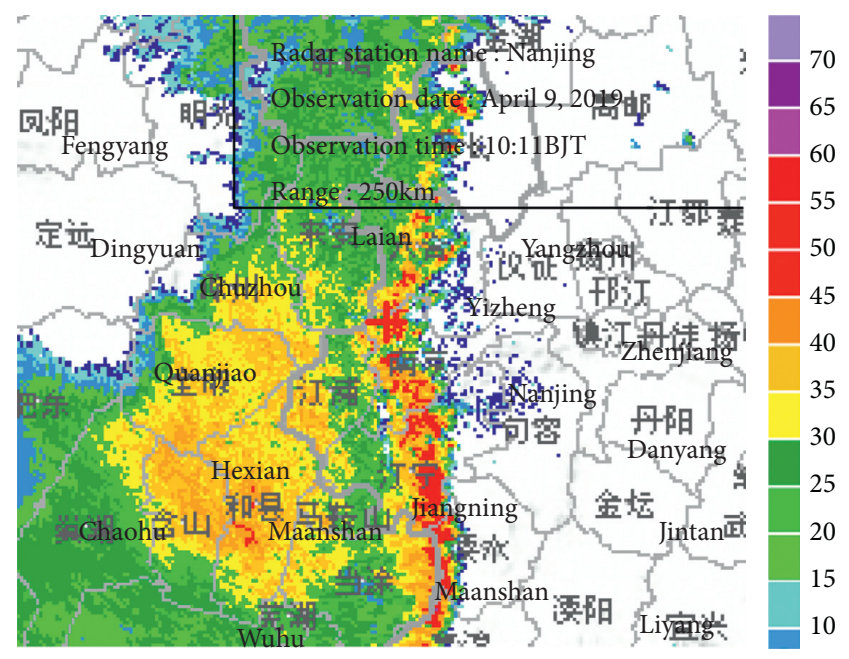

Figure 16: Radar map of Nanjing radar station at 10:11 on April 9, 2019.

perpendicular to the $z$-axis. At the moment, it is considered that the thunder source has occurred above the observation point. This experiment proves the validity of the calibration method well and gives real-time data feedback to the thunderstorm forecast warning.

6.2. Experiments in Fair Weather. On the same day, at 13:26 on April 9, 2019, the results measured by AEFA and microphone array are shown in Table 3. At this time, the radar map is shown in Figure 17.

In Table 3, the charge position obtained from the observation point is $(0.860,-52.32,21.54)$ (unit: $\mathrm{km}$, degrees, degrees). At this time, the thunderstorm charge is about 52.32 degrees south by west and $0.860 \mathrm{~km}$ away from the observation point. However, its elevation angle is relatively small, only reaching 21.54 degrees. This shows that, at about 13:26, according to the data shown in Table 3 , there is no thunderstorm cloud above the observation point. In Figure 17, the radar echo intensity at the observation point is only $15 \mathrm{dBZ}$, which means that there is no thunderstorm above the point because there is only a small amount of charge accumulation in the cloud. In short, at 13:26 on April 9, 2019, there is no thunderstorm cloud generated at the observation point, and the radar map data are consistent with the calibration method, once again verifying the effect of this method. Besides, combined with the data in Tables 2 and 3, it can be speculated that there is thunderstorm activity nearby and it may move further southward.
TABLE 3: Experimental results in fair weather.

\begin{tabular}{lc}
\hline Data source & Results \\
\hline Electric field components & $(0.056,-0.064,0.165)$ \\
AEFA angle & $(0.845,-48.81,21.21)$ \\
Observation angle & $(0.860,-52.32,21.54)$ \\
Microphone array & $(-0.0352,0.0174,-0.0136)$ \\
\hline
\end{tabular}

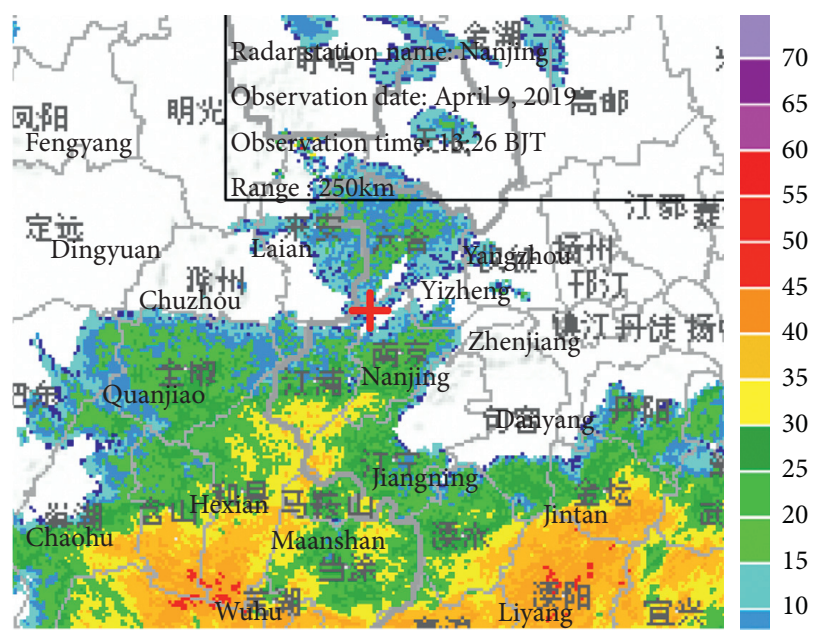

FIGURE 17: Radar map of Nanjing radar station at 13:26 on April 9, 2019.

At the same time point, we use Ref. [29] to locate the thunder source and compare it with the calibration method again. Similarly, the observation point we select is $(0.03,0.04$, -0.01 ) (units: $\mathrm{km}$ ), and the array element spacing is set to $0.8 \mathrm{~m}$. Table 3 shows the measured results.

In Table 3, from the array observation angle, the sound source point coordinates measured by the microphone array are $(-0.0352,0.0174,-0.0136)$ (units: $\mathrm{km}, \mathrm{km}, \mathrm{km}$ ). The sound source is about 26.29 degrees north by east, and it is only $0.042 \mathrm{~km}$ away from the observation point. In particular, the elevation angle is -19.09 degrees. This means that the sound source is below the $X O Y$ plane. In summary, there is no thunder source generated at the observation point at 13:26 on April 9, 2019. There is a good consistency between the measurement results of the microphone array, of the radar map, and of the results by the calibration method. Better application effects are once again verified.

6.3. Contrast Experiments. Firstly, it can be seen from Sections 6.1 and 6.2 that when the apparatus is in an open environment, the data obtained by the observation angle can be well matched with the real-time radar map and the microphone array. The results are still in radar range, and the measured data are relatively reliable.

Next, we select the experiments in thunderstorm weather, and the calibration method is compared with references [20,33]. Table 4 shows the contrast results.

As can be seen from Table 4, all of them can measure the vertical component. However, it is obvious that Ref. [33] cannot measure the horizontal component. The most direct 
TABLE 4: Comparison with references $[20,33]$.

\begin{tabular}{|c|c|c|c|c|c|}
\hline $\begin{array}{l}\text { Reference } \\
\text { source }\end{array}$ & $\begin{array}{l}\text { Horizontal electric } \\
\text { field component }\end{array}$ & $\begin{array}{l}\text { Vertical electric field } \\
\text { component }\end{array}$ & Position $M$ & Precision compensation & $\begin{array}{c}\text { Performance } \\
\text { analysis }\end{array}$ \\
\hline Paper & Equation (6) & Equation (6) & Tables 2 and 3 & Microphone array & Equation (10) \\
\hline Ref. [20] & Equations (18) and (19) & Equation (20) & - & $\begin{array}{c}\text { Particle swarm } \\
\text { optimization }\end{array}$ & - \\
\hline Ref. [33] & - & $0.637 \mathrm{kV} / \mathrm{m}$ & - & - & - \\
\hline
\end{tabular}

TABLe 5: Experimental results at 10:06 on April 9, 2019, from Ref. [21].

\begin{tabular}{lcc}
\hline Data source & $\left(E_{x}, E_{y}, E_{z}\right)$ & Coordinates $(\mathrm{km})$ \\
\hline N1 & $(-0.072,0.105,0.406)$ & $(0.094,-0.138,0.533)$ \\
N2 & $(-0.012,0.108,0.667)$ & $(0.085,-0.149,0.486)$ \\
N3 & $(-0.032,0.033,0.105)$ & $(0.102,-0.127,0.519)$ \\
\hline
\end{tabular}

reason is that they used a one-dimensional AEFA. In contrast, both this paper and Ref. [20] can simultaneously measure the $3 \mathrm{D}$ electric field components. In fact, after measuring the $3 \mathrm{D}$ electric field components, the charge position may not be obtained. On the one hand, there is a lack of models that can reflect the spatial relationship between the charge and AEFA. On the other hand, there is no further derivation of the charge localization formula. Compared with references [20,33], combined with Tables 2 and 3, this paper carries out the calibration work on the premise of charge localization, which has good continuity. In terms of precision compensation, unlike Ref. [20], the paper adopts an array-based sound source localization method, which can provide a data source for the distributed multisource thunderstorm monitoring system. Finally, based on the theory of indirect measurement, this paper studies the charge localization performance and further verifies the method. However, references [20,33] perform poorly in validation.

In fact, the calibration method can also be verified by using the data measured by several AEFA which are not in the same place. According to Section 5.1 of Ref. [21], the data shown in Table 5 can be obtained.

The data of apparatus $N 1, N 2$, and $N 3$ are given in Table 5. We assume that N1 is the main AEFA and N2 and $N 3$ are two different observation points. It can be seen from Ref. [21] that the positions of N2 and N3 are $(0,-1,0)$ and $(-1,1,0)$ (units: $\mathrm{km})$, respectively. Based on this, after using the calibration method, the thunderstorm charge positions at observation points $N 2$ and $N 3$ are $(0.094,0.862,0.533)$ and $(1.094,-1.138,0.533)$ (units: $\mathrm{km})$, respectively. At the same time, according to the electric field data measured by $N 2$ and $N 3$, the thunderstorm charge positions are $(0.085$, $0.851,0.486)$ and $(1.102,-1.127,0.519)$ (units: $\mathrm{km}$ ), respectively. In order to further compare the data obtained by the calibration method with the data measured by AEFA at the observation point, the ranging and direction-finding error rates between them are given in Table 6 . It should be noted that the Cartesian coordinate data have been
TABLE 6: Spherical coordinate data of thunderstorm charge.

\begin{tabular}{lcc}
\hline Data source & $N 2$ & $N 3$ \\
\hline Calibration method & $(1.018,83.78$, & $(1.666,-46.13$, \\
& $31.58)$ & $18.66)$ \\
AEFA data & $(0.984,84.30$, & $(1.659,-45.64$, \\
Ranging error & $29.61)$ & $18.22)$ \\
Direction-finding & $3.40 \%$ & $0.42 \%$ \\
error & Within $6.24 \%$ & Within $2.36 \%$ \\
\hline
\end{tabular}

transformed into the spherical coordinate data (unit: $\mathrm{km}$, degrees, degrees) shown in Table 6.

It can be seen from Table 6 that the errors of ranging and direction finding are within $3.40 \%$ and $6.24 \%$, respectively, which are acceptable. This not only verifies the validity of the calibration method but also reflects the better performance of AEFA.

The above experiments better reproduce the process from the generation of the thunderstorm cloud to their disappearance. The results of the calibration method are close to those of radar map and microphone array. In general, this method has a good effect.

\section{Conclusions}

The meteorological observation data have the characteristics of time series, and the number of these data is huge. The intellectualization of algorithms and equipment for observation is an indispensable part of the 4IR. Based on the 3D AEFA, we propose a calibration method of the thunderstorm charge localization. And then, from the observation point, a new coordinate system is established to conduct the charge localization calibration. Finally, the effectiveness of the method is verified by experiments in thunderstorm and fair weather. At the same time, the relevant references are selected and compared with the method, and the results are in good agreement.

The paper provides an effective solution to solve the problem that the one-dimensional AEFA can only measure a single electric field component. In addition to being used for thunderstorm detection, the proposed method can also ensure the importance of obtaining data from the observation angle. It should be noted that the existing research shows that the joint work of multiple devices can produce better results in 4IR, while the proposed method is based on a single AEFA. Therefore, whether the monitoring range and accuracy can be improved based on the network observation 
of thunderstorm with multiple devices will be carried out in the future.

\section{Data Availability}

The data used to support the findings of this study are available from the corresponding author upon request.

\section{Conflicts of Interest}

The authors declare that there are no conflicts of interest regarding the publication of this paper.

\section{Acknowledgments}

This research was supported by the National Key Research and Development Program of China (Grant nos. 2019YFE012990 and 2018YFC1506102), the National Natural Science Foundation of China (Grant no. 41605121), the Key Research and Development Plan of Jiangsu Province, China (Grant no. BE2018719), and the Advantage Discipline "Information and Communication Engineering" of Jiangsu Province, China, which are highly appreciated by the authors.

\section{References}

[1] W. Meng, "Thunderstorm cloud-to-ground lightning characteristics in the contiguous guangzhou city and the influences of local environmental conditions," Journal of Applied Meteorological Science, vol. 19, no. 5, pp. 611-619, 2008.

[2] A. Dai, "Global precipitation and thunderstorm frequencies. Part I: seasonal and interannual variations," Journal of Climate, vol. 14, no. 6, pp. 1092-1111, 2000.

[3] W. Wang and G. W. Bureau, "Protection measures of lightning electrostatic induction and lightning electromagnetic induction," Modern Architecture Electric, vol. 5, no. 5, pp. 45-48, 2014.

[4] M. Ishii, K. Miyabe, and A. Tatematsu, "Induced voltages and currents on electrical wirings in building directly hit by lightning," Electric Power Systems Research, vol. 85, pp. 2-6, 2012.

[5] S. Kaivonen and E. C.-H. Ngai, "Real-time air pollution monitoring with sensors on city bus," Digital Communications and Networks, vol. 6, no. 1, pp. 23-30, 2020.

[6] G. Jayendra, R. Lucas, S. Kumarawadu et al., "Intelligent lightning warning system," in Proceedings of the 2007 Third International Conference on Information and Automation for Sustainability, IEEE, Melbourne, Australia, December 2008.

[7] H. Xing, G. He, and X. Ji, "Analysis on electric field based on three dimensional atmospheric electric field apparatus," Journal of Electrical Engineering and Technology, vol. 13, no. 4, pp. 1696-1703, 2018.

[8] R. E. Hood, D. J. Cecil, F. J. Lafontaine et al., "Classification of tropical oceanic precipitation using high-altitude aircraft microwave and electric field measurements," Journal of the Atmospheric Sciences, vol. 63, no. 1, pp. 218-233, 2006.

[9] X. Su, K. Fan, and W. Shi, "Privacy-preserving distributed data fusion based on attribute protection," IEEE Transactions on Industrial Informatics, vol. 15, no. 10, pp. 5765-5777, 2019.

[10] G. B. Burns, B. A. Tinsley, A. V. Frankkamenetsky, O. A. Troshichev, W. J. R. French, and A. R. Klekociuk, "Monthly diurnal global atmospheric circuit estimates derived from vostok electric field measurements adjusted for local meteorological and solar wind influences," Journal of the Atmospheric Sciences, vol. 69, no. 6, pp. 2061-2082, 2012.

[11] A. I. Boldyrev, A. E. Vyazilov, V. N. Ivanov et al., "A highly sensitive field mill for registering weak and strong variations of the electric-field intensity of the Earth's atmosphere," Instruments and Experimental Techniques, vol. 59, no. 5, pp. 740-748, 2016.

[12] A. Fort, M. Mugnaini, V. Vignoli et al., "Design, modeling, and test of a system for atmospheric electric field measurement," IEEE Transactions on Instrumentation and Measurement, vol. 60, no. 8, pp. 2778-2785, 2011.

[13] C. Schumann, M. M. F. Saba, R. B. G. da Silva, and W. Schulz, "Electric fields changes produced by positives cloudto-ground lightning flashes," Journal of Atmospheric and Solar-Terrestrial Physics, vol. 92, pp. 37-42, 2013.

[14] A. V. Frank-Kamenetsky, O. A. Troshichev, G. B. Burns, and V. O. Papitashvili, "Variations of the atmospheric electric field in the near-pole region related to the interplanetary magnetic field," Journal of Geophysical Research: Space Physics, vol. 106, no. A1, pp. 179-190, 2001.

[15] R. Vishnu R, V. A. Kumar, T. S. Sreekanth et al., "Formation of thunderclouds in a region of high lightning incidence, inferred from AWS, ceilometer and an electric field mill," in Proceedings of the 7th Asia-Pacific International Conference on Lightning, IEEE, Chengdu, China, November 2011.

[16] P. Baranski, M. Loboda, J. Wiszniowski, and M. Morawski, "Evaluation of multiple ground flash charge structure from electric field measurements using the local lightning detection network in the region of Warsaw," Atmospheric Research, vol. 117, pp. 99-110, 2012.

[17] H. Xing and X. Yang, "Sound source localization fusion algorithm and performance analysis of a three-plane fiveelement microphone array," Applied Sciences, vol. 9, no. 12, 2019.

[18] F. Moghaddam, P. Wieder, R. Yahyapour et al., "A multilayered policy generation and management engine for semantic policy mapping in clouds," Digital Communications and Networks, vol. 6, no. 1, pp. 38-50, 2020.

[19] X. Wen, "Measuring method of three dimensional atmospheric electric field based on coplanar decoupling structure," Journal of Electronics \& Information Technology, vol. 36, no. 10, pp. 2504-2508, 2014.

[20] W. Xu, C. Zhang, X. Ji et al., "Inversion of a thunderstorm cloud charging model based on a 3D atmospheric electric field," Applied Sciences, vol. 8, no. 12, 2018.

[21] X. Yang and H. Xing, "A data complementary method for thunderstorm point charge localization based on atmospheric electric field apparatus array group," Digital Communications and Networks, 2020.

[22] H. Xing, X. Yang, and J. Zhang, "Thunderstorm cloud localization algorithm and performance analysis of a threedimensional atmospheric electric field apparatus," Journal of Electrical Engineering and Technology, vol. 14, no. 6, 2019.

[23] S. Dyasti, E. Hagras, and H. EI-Hennawy, "QSM-IDM-a novel quadrature spatial modulation based on interleaving division multiplexing for multiple antenna system," Digital Communications and Networks, vol. 5, no. 3, pp. 183-188, 2019.

[24] X. Ji, H. Xing, and W. Xu, "The relation of three dimension atmospheric electric field and thundercloud charge position," Insulators and Surge Arresters, vol. 6, pp. 63-68, 2015.

[25] X. Yang, H. Xing, W. Xu et al., "A moving path tracking method of the thunderstorm cloud based on the threedimensional atmospheric electric field apparatus," Journal of Sensors, vol. 2021, Article ID 8856033, 13 pages, 2021. 
[26] M. Fujii, M. Yamada, M. Fukuma et al., "Relationship between deterioration of epoxy resin and change of space charge distribution under nonuniform electric field," Electronics \& Communications in Japan, vol. 101, 2018.

[27] Y.-J. Li, G.-S. Zhang, J. Wen et al., "Spatial and temporal evolution of a multi-cell thunderstorm charge structure in coastal areas," Chinese Journal of Geophysics, vol. 55, no. 5, pp. 498-508, 2012.

[28] F. Leberl, "Introduction to the mathematics of inversion in remote sensing and indirect measurement," Tectonophysics, vol. 65, no. 3-4, pp. 376-378, 1980.

[29] X. Yang, H. Xing, and X. Ji, "Sound source omnidirectional positioning calibration method based on microphone observation angle," Complexity, vol. 2018, Article ID 2317853, 15 pages, 2018.

[30] C. Quested, A. Moorhouse, B. Piper, and B. Hu, “An analytical model for a dodecahedron loudspeaker applied to the design of omni-directional loudspeaker arrays," Applied Acoustics, vol. 85, pp. 161-171, 2014.

[31] X. Su, G. Sperli, V. Moscato, A. Picariello, C. Esposito, and C. Choi, "An edge intelligence empowered recommender system enabling cultural heritage applications," IEEE Transactions on Industrial Informatics, vol. 15, no. 7, pp. 4266-4275, 2019.

[32] M. Lahby, A. Essouiri, and A. Sekkaki, “A novel modeling approach for vertical handover based on dynamic k-partite graph in heterogeneous networks," Digital Communications and Networks, vol. 5, no. 4, pp. 297-307, 2019.

[33] H. Wan, G. Wei, Y. Cui et al., "Influence factor analysis of atmospheric electric field monitoring near ground under different weather conditions," Journal of Physics: Conference Series, vol. 418, no. 1, p. 2029, 2013. 